\title{
Enfermedad del coronavirus 2019: la importancia del distanciamiento social
}

\author{
Coronavirus disease 2019: the importance of social distancing \\ Doença de coronavírus 2019: a importância do distanciamento social \\ Fernando Antonio Ignacio González
}

\section{RESUMEN}

La actual pandemia de la enfermedad de coronavirus 2019 constituye una severa amenaza para las personas y la economía a nivel global. La adopción de medidas tendientes a reducir la velocidad de expansión de la pandemia es fundamental. En este trabajo se busca examinar el potencial impacto de la implementación de medidas de distanciamiento social. En particular, a partir de una reducción en la tasa de reproducción $\left(R_{0}\right)$ se examina su impacto sobre la dinámica de infectados y fallecidos.

Se recurre a la utilización de una extensión del modelo SIR estándar, el cual considera explícitamente a aquellas personas infectadas pero que aún no pueden transmitir la enfermedad (expuestos) y aquellos que han fallecido (fallecidos) y una tasa de reproducción decreciente en el tiempo. Este comportamiento de la tasa de reproducción reflejaría la implementación de medidas de distanciamiento a partir de diferentes especificaciones.

Los resultados sugieren que las medidas de distanciamiento contribuyen a reducir significativamente la cantidad máxima de infectados activos y fallecidos diarios. Esto es, logran aplanar las curvas de contagios y fallecimientos.

Palabras claves: Pandemia; Distanciamiento social; Coronavirus.

\section{ABSTRACT}

The current coronavirus disease 2019 pandemic poses a severe threat to people and the economy globally. The adoption of measures to reduce the speed of expansion of the pandemic is essential. This paper seeks to examine the potential impact of implementing social distancing measures. In particular, from a reduction in the reproduction rate $\left(R_{0}\right)$ its impact on the dynamics of infected and deceased is examined.

The use of an extension of the standard SIR model is used, which explicitly considers those infected but not yet able to transmit the disease (exposed) and those who have died (deceased) and a decreasing rate of reproduction over time. This behavior of the reproduction rate would reflect the implementation of distance measurements based on different specifications.

The results suggest that the distancing measures contribute to significantly reduce the maximum number of active infected and daily deceased. That is, they manage to flatten the contagion and lethality curves.

Keywords: Pandemic; Social distancing; Coronavirus.

\section{RESUMO}

A atual pandemia da doença de coronavírus 2019 constitui uma severa ameaça para as pessoas e a economia a nível global. A adoção de medidas para reduzir a velocidade de expansão da pandemia é essencial. Neste trabalho se busca examinar o impacto potencial da implementação de medidas de distanciamento social. Em particular, se examina o impacto, na dinâmica de infectados e óbitos, de uma redução na taxa de reprodução $\left(R_{0}\right)$.

Se recorre a utilização de uma extensão do modelo SIR padrão o qual considera explicitamente os infectados que ainda não podem transmitir a doença (expostos) e os que morreram (falecidos) e uma taxa de reprodução decrescente ao longo do tempo. Esse comportamento da taxa de reprodução refletiria a implementação de medições de distância com base em diferentes especificações.

Os resultados sugerem que as medidas de distanciamento contribuem para reduzir significativamente a quantidade máxima de infectados ativos e falecidos diariamente. Isto é, eles conseguem achatar as curvas de contágios e mortes.

Palavras-chave: Pandemia; Distanciamento social; Coronavirus.

1. Instituto de Investigaciones Económicas y Sociales del Sur, Universidad Nacional del Sur (UNS)-CONICET, Bahía Blanca, Argentina.

$\triangle$ Instituto de Investigaciones Económicas y Sociales del Sur. San Andrés 800, Campus Palihue, Bahía Blanca, Argentina. fernando_gonzalez01@hotmail.com | Recebido em: 05/05/2020 | Aprovado em: 11/08/2020 


\section{INTRODUCCIÓN}

La actual pandemia de la enfermedad de coronavirus 2019 (COVID-19) ha afectado profundamente la rutina diaria de millones de personas. Este desastre natural de tipo biológico, cuyo origen se remonta a la ciudad china de Wuhan y a fines del 2019, es causado por el síndrome respiratorio agudo grave coronavirus 2 (SARS-CoV-2). Globalmente, se han producido más de 17.8 millones de contagios y más de 680 mil muertes ${ }^{1}$. Más de un quinto de la población global cumple algún tipo de cuarentena, a la vez que numerosos países intentan aprobar paquetes de estímulo económico -el Congreso de Estados Unidos ha aprobado de un paquete por U\$S 2.2 billones- para mitigar el impacto socio-económico de la pandemia.

Predecir las consecuencias macroeconómicas de esta pandemia es una tarea compleja. Estados Unidos reporta datos con alta frecuencia y anticipa un significativo impacto negativo: únicamente durante marzo la cantidad de personas desempleadas aumentó en 5.7 millones ( +0.9 punto porcentual) ${ }^{2}$. Explotando variaciones geográficas en la incidencia del COVID-19, Béland et al. ${ }^{3}$ demuestran que este aumento es consecuencia de la pandemia. También hallan que las personas más afectadas son las de bajo nivel educativo o que se desempeñan en ocupaciones que no se pueden realizar en forma remota. A nivel global, se espera una caída de 2 billones en los ingresos y que los países más afectados sean los exportadores de commodities y aquellos con vínculos comerciales más estrechos con los países más afectados por la pandemia ${ }^{4}$. Por primera vez desde 1998 , se espera un incremento de la pobreza a nivel global ${ }^{5}$. Igual pronóstico es esperable en términos de la desigualdad ${ }^{6}$.

La pandemia parece expandirse a diferentes velocidades en distintos países. La cantidad de fallecimientos, reportada en el Gráfico 1, indica que Europa occidental, Norteamérica y América del Sur son las regiones más afectadas. Los países en desarrollo, en particular, presentan una mayor vulnerabilidad: sus sistemas sanitarios poseen una menor capacidad de respuesta, a la vez que sus gobiernos cuentan con una menor capacidad de asistencia y estímulo fiscal.

Los países del Mercado Común del Sur (MERCOSUR) no escapan a esta realidad. Todos ellos han confirmado infectados y fallecidos por COVID-19* -esto es especialmente cierto para Brasil, país que lidera ambas series en la actualidad con más de 12000 infectados y 400 fallecimientos, por millón de habitantes-. Además, estos países han dispuesto algún tipo de medida tendiente a fomentar el distanciamiento social ${ }^{* *}$ y achatar la curva de contagios, de modo de evitar un colapso en sus sistemas de salud. Paraguay dispuso medidas de distanciamiento social generalizado y obligatorio desde el 20 de marzo -aunque desde el 11 de marzo fue restringida la circulación y asistencia a eventos masivos-. Argentina también implementó tempranamente (20 de marzo) un esquema de distanciamiento generalizado y obligatorio. Uruguay ha impuesto restricciones menos severas (declaración de emergencia sanitaria desde el 13 de marzo) como el aislamiento, únicamente, de personas con antecedentes de visita a países de riesgo y cierre de las fronteras. Brasil parece distanciarse de los casos anteriores al no definir una estrategia coordinada a nivel nacional. La provincia de San Pablo (24 de marzo) ha sido uno de los primeros estados en implementar medidas de distanciamiento generalizado y obligatorio. Otros estados han tomado medidas similares.

Simultáneamente, estos países han implementado distintas acciones de política fiscal - monetaria ${ }^{8}$. Paraguay redujo la alícuota del Impuesto al Valor Agregado y eliminó aranceles para insumos médicos, impulsó un paquete de medidas de emergencia equivalente a $2.5 \%$ del PBI y su autoridad monetaria ha reducido la tasa de interés de referencia (-175 puntos básicos). Argentina ha adoptado medidas cuyo costo asciende a $1.2 \%$ del PBI (mayores transferencias a hogares pobres, aumento gasto en salud, subsidios al pago de salarios, facilidades crediticias). Por su parte, Uruguay ha anunciado medidas equivalentes al $0.7 \%$ de su PBI (beneficios impositivos y previsionales, trasferencias a hogares pobres y desempleados, etc.) y su autoridad monetaria

La cantidad de casos confirmados y fallecimientos son presentados en el Anexo 1.

Por distanciamiento social se entiende a aquel conjunto de medidas tendientes a reducir la velocidad de transmisión del brote, a partir de una disminución del contacto físico entre personas ${ }^{7}$. Puede contemplar medidas focalizadas (a subgrupos poblacionales o áreas geográficas) o generalizadas. 
anunció reducciones en los requerimientos de encaje. Finalmente, el gobierno de Brasil anunció la implementación de medidas equivalentes al $6.25 \%$ del PBI (incluye transferencias a hogares pobres, desempleados, exención impositiva para la importación de insumos médicos, transferencias del gobierno central a las provincias y municipios, etc.). Su autoridad monetaria recortó su tasa de referencia ( -50 puntos básicos) y redujo los requerimientos de encaje.

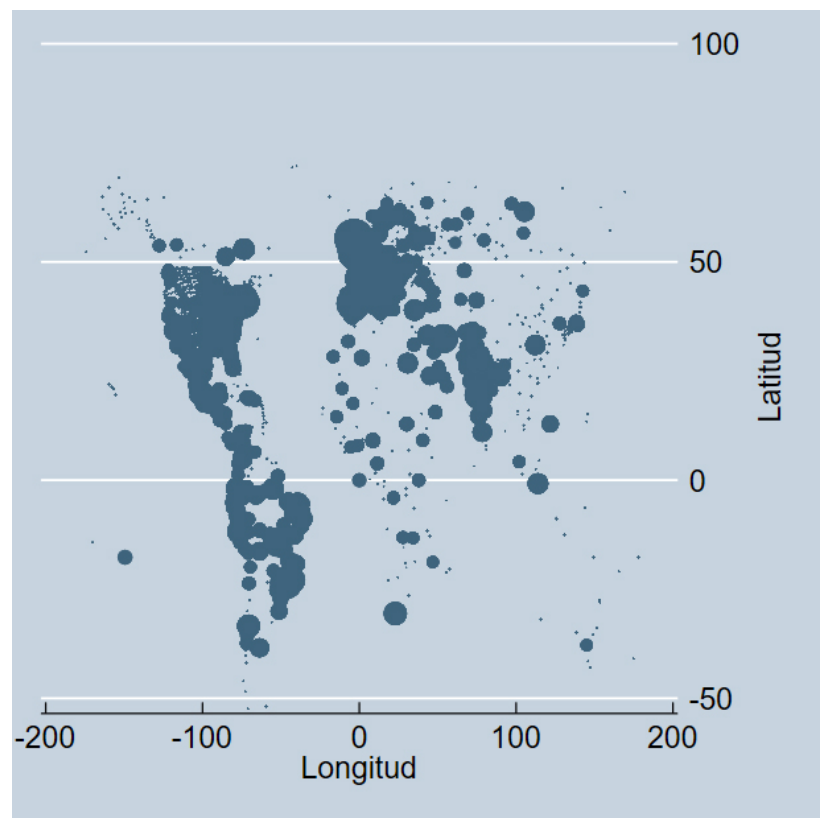

Gráfico 1: Distribución espacial de fallecimientos por COVID-19 Fuente: elaboración propia en base a Johns Hopkins University ${ }^{1}$ Nota: se incluyen los registros publicados hasta el 2 de agosto de 2020 inclusive. El tamaño del marcador se asocia a la cantidad de fallecimientos.

Un extenso debate se ha generado en torno a la eficacia de las medidas de distanciamiento para contener la pandemia. Intuitivamente, al reducir la cantidad de contactos diarios con otras personas, se espera una disminución en la velocidad de propagación del virus. Sin embargo, la evidencia es escasa en términos de revelar en qué medida se logra aplanar la curva de infectados y fallecidos o cuánto tiempo resulta necesario para observar resultados. Estas son cuestiones relevantes para los hacedores de política: imponer amplias medidas de distanciamiento generalizado, asumiendo su elevado costo social y económico, pero cuyos efectos en términos de aplanar la curva de infectados sea mínimo podría indicar que otra estrategia de contención es conveniente.

En este contexto, en el presente trabajo se busca analizar el potencial impacto de las medidas de distanciamiento social en la evolución de la pandemia de COVID-19. En particular, se hace uso de una variante del modelo epidemiológico SIR de Kermack y McKendrick $^{9}$ que permite un mayor grado de desagregación en subconjuntos poblacionales y contempla una tasa de contagio decreciente en el tiempo. Este tópico es especialmente relevante dada la aparente existencia de un trade-off entre salud y economía. Aquellos países que inician la pandemia con peores indicadores socio-económicos poseen un menor margen de maniobra. Este es el caso de Argentina que inicia 2020 con una incidencia de la pobreza multidimensional cercana al $40 \%{ }^{10}$ y un reducido espacio fiscal ${ }^{11}$. La existencia de profundas disparidades regionales ${ }^{12,13}$ puede constituir un agravante de lo anterior.

La sección 2 describe la metodología y fuentes de información empleadas. La sección 3 presenta los principales resultados $y$, finalmente, la sección 4 discute las principales conclusiones.

\section{METODOLOGÍA Y FUENTES DE INFORMACIÓN}

En Epidemiología, típicamente, se emplea alguna variante del modelo SIR (Susceptibles, Infectados, Recuperados) para modelar la evolución temporal de un brote infeccioso. En particular, una extensión frecuentemente empleada es el denominado modelo SEIRD (Susceptibles [S], Expuestos $[E]$, Infectados $[\mathrm{I}]$, Recuperados [R], Fallecidos $[D])$ que desagrega entre aquellas personas que han sido infectadas, pero aún no pueden transmitir la enfermedad (Expuestos) y aquellas que han dejado de pertenecer al grupo de Infectados -ya sea por recuperación o fallecimiento-. La dinámica del brote es definida a partir del siguiente sistema de ecuaciones:

$$
\begin{gathered}
\dot{S}=-\beta I \frac{S}{N} \\
\dot{E}=\beta I \frac{S}{N}-\alpha E
\end{gathered}
$$




$$
\begin{gathered}
\dot{I}=\alpha E-(1-\varnothing) \gamma I-\varnothing * \rho^{*} I \\
\dot{R}=(1-\varnothing) \gamma I \\
\dot{D}=\varnothing * \rho^{*} I \\
N=S+E+I+R+D
\end{gathered}
$$

Donde las ecuaciones 1 a 5 modelan la dinámica temporal de cada subgrupo del modelo. $\beta$ es la tasa de contagio diaria promedio, $\alpha$ es la inversa del período de incubación promedio en días, $\gamma$ es la inversa del período de infección (días durante los cuales una persona infectada puede transmitir la enfermedad) ${ }^{* * *}, \rho$ es la inversa del período de letalidad (días desde la infección hasta el fallecimiento) y $\varnothing$ es la tasa de letalidad de los infectados. A partir de los parámetros $\beta$ y $\gamma$ es posible calcular la tasa de reproducción $\left(R_{0}\right)$ :

$$
R_{0}=\frac{\beta}{\gamma}
$$

La tasa de reproducción indica la cantidad de personas que, en promedio, cada individuo infectado contagia. Esta es una variable clave en la evolución de la pandemia. Tal como está definida en la ecuación $7, R_{0}$ es constante en el tiempo. Lógicamente, esto parece un supuesto poco realista considerando la implementación de medidas de distanciamiento social. Al respecto, se recurre a dos especificaciones alternativas de $R_{0}$.

Primero, asumiendo la implementación de una medida de distanciamiento social obligatorio y generalizado en el día $t_{i}$ que reduce la tasa de contagio $(\beta)$, se tiene:

$$
R_{0}=\left\{\begin{array}{l}
R_{0_{1}} \text { si } t<t_{i} \\
R_{0_{2}} \text { si } t \geq t_{i}
\end{array}\right.
$$

Con $\mathrm{R}_{0_{1}}>\mathrm{R}_{0_{2}}$. Esto implica una reducción discreta, de una única vez, en la tasa de reproducción

También puede concebirse como la tasa de recuperación, esto es, cada día una proporción 1/ de los infectados abandona este grupo y alcanza el siguiente estadio (fallecimiento o recuperado). al implementarse las medidas de distanciamiento. En adelante, a esta especificación se la denomina modelo discreto. Alternativamente, es posible considerar una reducción gradual y sostenida en la tasa de reproducción. Esto refleja el hecho que las medidas de distanciamiento se implementan en forma escalonada entre regiones o sectores:

$$
R_{0}=\frac{R_{0_{\text {inicial }}} R_{0_{\text {final }}}}{1+e^{-k\left(-x+x_{0}\right)}}+R_{0_{\text {final }}}
$$

Donde $R_{\text {oinicial }}$ es el $R_{0}$ en el primer día, $R_{0 \text { final }}$ es el $\mathrm{R}_{0}$ del último día, $k$ es la tasa de crecimiento logístico y $x_{0}$ es el valor $x$ del punto medio de la sigmoide En este caso, se asume que el $R_{0}$ sigue una distribución logística. Esta especificación se denominará, en adelante, modelo continuo. Debe resaltarse que, dadas las dificultades para estimar el parámetro $\beta$, típicamente se utiliza como input en estos modelos la medida $R_{0}$ y se despeja $\beta$.

Al igual que el $R_{0}$, es concebible que la tasa de letalidad $(\varnothing)$ no sea constante en el tiempo o entre sub-grupos poblacionales. Esta tasa puede depender de la edad de la persona y de la saturación del sistema de salud (una mayor cantidad de infectados aumenta las chances de que una persona adicional no pueda recibir atención médica). Entonces:

$$
\varnothing_{t}=m * \frac{I_{t}}{N}+\varnothing_{\text {base }}
$$

Donde $\varnothing_{\text {base }}$ es la tasa de letalidad base cuando existen pocos infectados. A medida que la proporción de infectados -sobre el total poblacional de referencia- aumenta, la letalidad también se incrementa. El parámetro $m$ refleja esto. La letalidad base resulta de un promedio ponderado de la letalidad por sub-grupo etario:

$$
\varnothing_{\text {base }}=\frac{g 1}{N_{0-19}} \varnothing_{g 1}+\frac{g 2}{N_{20-59}} \varnothing_{g 2}+\frac{g 3}{N_{60+}} \varnothing_{g 3}
$$

Lógicamente la letalidad por sub-grupo etario puede ser estimada a partir de múltiples formas funcionales. Aquí se implementa una forma sencilla e 
intuitiva, aunque, quizás, resulte en una sobre-simplificación. Otras especificaciones pueden incorporan la proporción de hombres en la población dada que estos parecen tener mayor riesgo de mortalidad por COVID-19 14 .

\section{Definición de parámetros}

Cada uno de los parámetros del modelo debe ser definido desde el día inicial. Esto resulta, sin dudas, problemático dado que el brote es reciente y las estadísticas de contagios y letalidad difundidas por cada país no son estrictamente comparables. Ventajosamente, la rápida respuesta de los investigadores permite contar con estimaciones preliminares. Al respecto, se sigue las propuestas de dos estudios que han tenido amplia difusión ${ }^{15,16}$. El $R_{0}$ empleado en estos casos oscila entre 2.4 y 2.25-2.75 15 16. La inversa del período de infección $(\gamma)$ se define en $1 / 4.5$ y la inversa del período de letalidad $(\rho)$ en $1 / 17^{16}$. La tasa de letalidad promedio de los infectados $(\varnothing)$ es definida en $0.9 \%$ en Ferguson et al. ${ }^{15}$ quienes, ventajosamente, también presentan estimaciones desagregadas por rango etario -las cuales son empleadas en adelante- ${ }^{* * * *}$. La inversa del período de incubación $(\alpha)$ es definida en $1 / 4^{15}$.

Lógicamente, con el transcurso del tiempo se podrán obtener mejores estimaciones de cada etapa de la pandemia. A su vez, diferentes modelos pueden emplear distintos valores para estos parámetros, deviniendo en resultados disímiles. En particular, en el caso del $R_{0}$, se han utilizado otros valores para este parámetro: 3.8 para los inicios de la pandemia en China ${ }^{17}, 3.25$ para el caso de Italia ${ }^{18}$ o 2.28 para el caso del crucero Diamond Princess ${ }^{19}$.

\section{RESULTADOS}

En primer lugar, se presentan las estimaciones del modelo SEIRD que surgen de simular un escenario con características similares al caso de Argentina. Esto implica una población ( $\mathrm{N})$, estimada

\footnotetext{
**** Para el grupo 0-19 años es de 0.004\%, para el grupo 20-59 es de $0.215 \%$ y para los mayores de 60 es de $5.53 \%$. Estos valores constituyen promedios simples de intervalos dentro de cada grupo.
}

para 2020, de 45.38 millones de habitantes ${ }^{20}$. Las participaciones poblacionales por rango etario son de $32.08 \%$ (0-19 años), $52.21 \%$ (20 a 59) y $15.71 \%$ (60 y más).

El gráfico 2 presenta la dinámica de la epidemia -en términos de infectados y fallecidos- considerando el modelo discreto. Se asume un $\mathrm{R}_{0}$ inicial de 3 y un $R_{0}$ final de 0.9. Esta reducción es consistente con la disminución de la movilidad observada entre marzo y abril de 2020 luego de la implementación de las medidas de distanciamiento obligatorio ${ }^{21}$. Se incorporan tres especificaciones distintas según cuántos días transcurren desde el primer caso confirmado hasta la implementación de medidas (60, 53 ○ 67 días). Lógicamente, otras variantes son concebibles (con medidas anteriores o posteriores). Esto, sin embargo, es suficiente para evidenciar la importancia de su implementación.

Se puede observar que las dinámicas difieren considerablemente en estos casos. Esto es, la implementación temprana de medidas de distanciamiento social altera persistentemente a la dinámica de la epidemia. En el caso de los infectados, el pico de la pandemia alcanza a 5400 personas con la implementación más temprana (día 53) pero a 30400 en la implementación tardía (equivalente a un $83 \%$ de reducción). Esta no es una cuestión menor dado que puede significar la saturación del sistema de salud: es decir, la cantidad de infectados que requieren algún tipo de hospitalización es mayor a la cantidad de pacientes que pueden ser atendidos, en cada momento del tiempo. Lógicamente, esto aumenta el riesgo de mortalidad para los no atendidos.

En términos de fallecimientos, la dinámica es similar. La implementación temprana de medidas de distanciamiento achata la curva. La cantidad de fallecimientos acumulados pasa de 275 a 1477 al considerar la implementación más temprana y la más tardía (reducción de $82 \%$ ). Resultados similares surgen de variar la intensidad del distanciamiento social (reducción del $\mathrm{R}_{0}$ ) en lugar de cambios en el momento de su implementación: esto es, medidas de distanciamiento más agresivas pero implementadas luego de una mayor cantidad de días desde el primer caso pueden generar una dinámica similar a la que surge de medidas menos severas de distanciamiento, pero implementadas con mayor anticipación. 

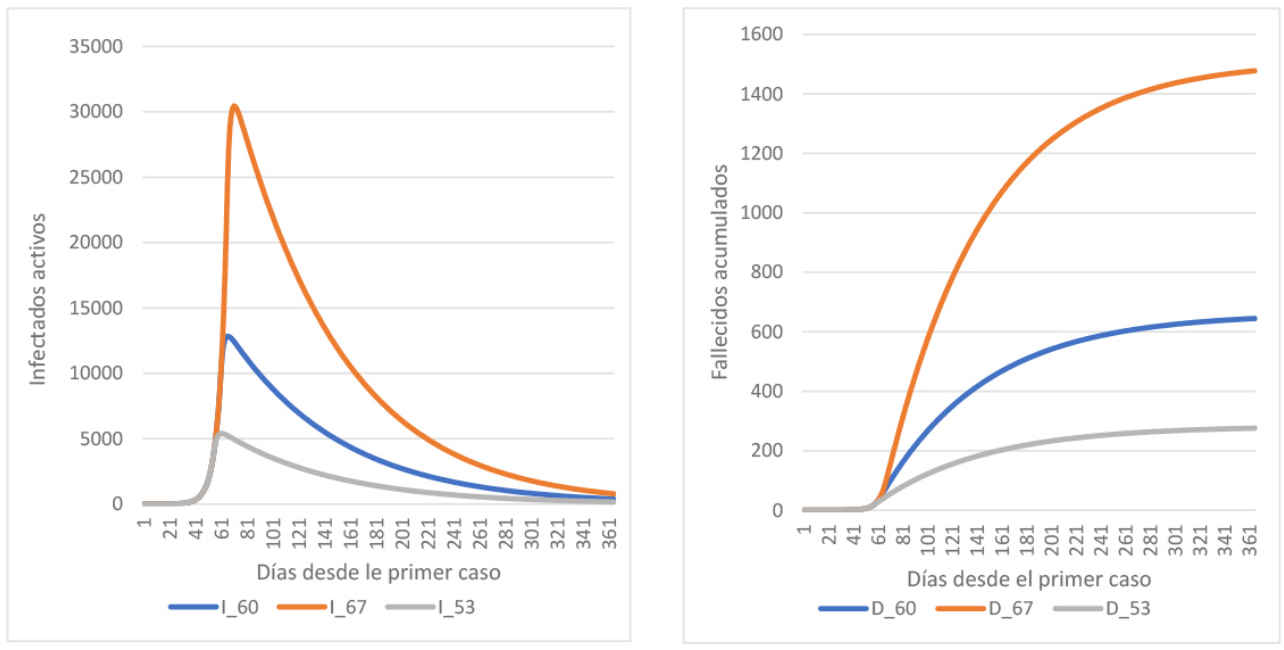

Gráfico 2: Infectados y fallecidos para distintos momentos de implementación de distanciamiento Fuente: elaboración propia

Lo anterior, sin embargo, es problemático en el actual contexto de la pandemia de COVID-19. En primer lugar, los hacedores de política no cuentan con información para amplios períodos de tiempo, sino que apenas de algunas pocas semanas. Esto incorpora una gran incertidumbre en los parámetros de cualquier modelo que intente reflejar la dinámica de la epidemia. En particular, la cantidad de contagios confirmados puede estar subestimando la verdadera cantidad de infectados $y$, entonces, generar un indebido retraso en la implementación de medidas de distanciamiento. Esta subestimación tiene lugar, dependiendo de cada país, a partir de la definición de caso sospechoso.

El concepto de caso sospechoso determina a qué personas se les realizarán los tests y varía entre países y en el tiempo para un mismo país. En el caso argentino, el Ministerio de Salud $^{22}$ consideró, en un principio, como sospechoso a aquellos casos de individuos con síntomas (fiebre más tos, odinofagia -dolor de garganta- o dificultad respiratoria) y antecedentes de viaje a países de riesgo en los 14 días previos -o haber estado en contacto con casos confirmados-. Posteriormente la definición de caso sospechoso se amplió al incluir a aquellas personas residentes o que hayan visitado áreas donde existe transmisión local, comunitaria o por conglomerado, del virus (Área Metropolitana de Buenos Aires, Chaco, Santa Fe, Ciudad de Córdoba, Alta Gracia, Rio Cuarto y Ushuaia) $)^{* * * * *}$. Al respecto, mientras más estrecha sea la definición de casos sospechoso menos tests serán realizados y mayor podrá ser la sub-estimación de los infectados.

En segundo lugar, aun cuando se cuente con información precisa del número de infectados a un momento de tiempo, la velocidad de los nuevos contagios (R0) puede aumentar rápidamente. En efecto, si además la implementación de medidas de distanciamiento no es instantánea, esto puede devenir en la saturación de la capacidad del sistema de salud. Por ello, las recomendaciones de política instan a una temprana implementación de medidas de distanciamiento?.

Por otra parte, el gráfico 3 presenta los resultados correspondientes al modelo continuo. Las diferentes especificaciones provienen de variaciones en la tasa de reducción del $\mathrm{R}_{0}$ y refleja distintas velocidades en la implementación de las medidas de distanciamiento. Se consideran tasas de reducción del $R_{0}$ de $0.02,0.018$ y 0.022 .

\footnotetext{
***** Los cambios en la definición de caso sospechoso pueden ser consultados en https://www.argentina.gob.ar/salud/ coronavirus-COVID-19/definicion-de-caso
} 

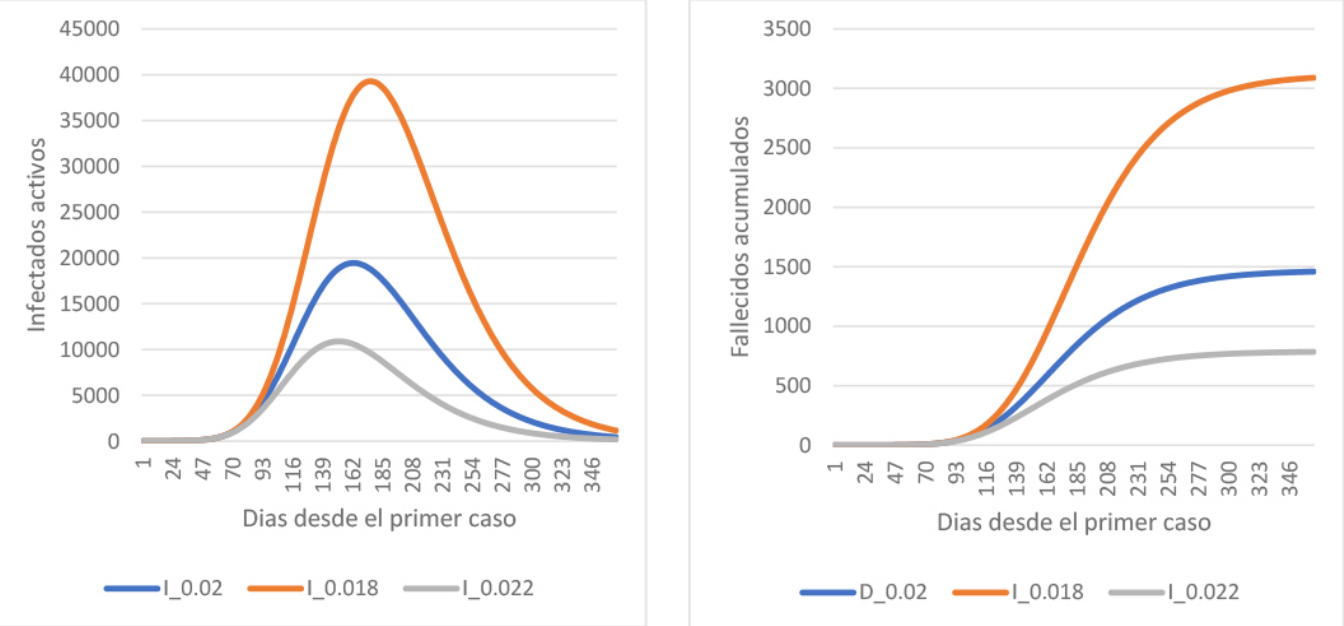

Gráfico 3: Infectados y fallecidos para distintas velocidades de tasa de reducción en modelo continuo Fuente: elaboración propia

Con este modelo, se observa una dinámica más suavizada en el tramo ascendente de la curva de infectados. Esto es esperable dada la disminución gradual pero sostenida en la tasa de reproducción. Los resultados aquí reportados confirman lo previamente discutido: las medidas de distanciamiento social pueden tener un significativo efecto en la dinámica de la pandemia.

La reducción gradual en la tasa de reproducción puede reflejar el hecho que no solo las autoridades gubernamentales pueden influir en las decisiones de movilidad y contacto de las personas -a partir de medidas de distanciamiento obligatorias- sino que éstas pueden, gradualmente, reducir su exposición a medida que la preocupación social por la pandemia aumenta. Al respecto, Chudik et al. ${ }^{23}$ modelan el distanciamiento social obligatorio y voluntario (este último dependiente de la probabilidad percibida de contagio). Andersen ${ }^{24}$ provee evidencia empírica de la magnitud del distanciamiento voluntario para el caso de Estados Unidos.

En síntesis, los resultados aquí reportados van en línea con lo sugerido con la evidencia internacional previa. Así, Milne y $\mathrm{Xie}^{25}$ proveen evidencia de la efectividad de las medidas de distanciamiento para el caso de Australia y hallan una reducción significativa en la cantidad acumulada de infectados $(66 \%$ a $24 \%$ en su mejor estimación) y del pico de infectados (hasta $80 \%$ al comparar diferentes especificaciones de distanciamiento). Estos resultados son robustos aun cuando las medidas son implementadas tardíamente. Ferguson et al. ${ }^{15}$ hallaron, para el caso de Reino Unido, que diferentes las medidas de distanciamiento pueden reducir la cantidad acumulada de muertes un $50 \%$ y disminuir el pico de demanda de hospitalización en $67 \%$. Para el caso de Brasil, Bastos y Cajueiro ${ }^{26}$ reportan que diferentes medidas de distanciamiento pueden reducir en un $50 \%$ el pico de infectados (equivalente en su estimación a 30 millones de personas).

\section{CONCLUSIONES}

El presente trabajo constituyó un aporte al debate acerca de la importancia de la temprana implementación de medidas de distanciamiento social. A partir de un modelo SEIRD (Susceptibles, Expuestos, Infectados, Recuperados, Fallecidos) se simuló la dinámica del brote de COVID-19. Los resultados dan cuenta de la importancia de las medidas de distanciamiento en aplanar la curva de infectados y fallecimientos. Esto es de suma importancia dada la limitada capacidad de respuesta de los sistemas de salud -especialmente entre países en desarrollo-.

En un contexto de amplia incertidumbre respecto a la verdadera velocidad de contagio y letalidad de la enfermedad, la mejor estrategia parece estar dada por la temprana implementación de medidas tendientes a la reducción de la tasa de 
reproducción. Lógicamente, esto no solo impone consecuencias epidemiológicas sino también económicas y sociales. La prohibición de circular impide la realización de una amplia cantidad de actividades. Esto acarrea una pérdida de ingresos y puestos de trabajos. En efecto, se espera una significativa caída en el producto global ${ }^{27}$.

Ante la existencia de un aparente trade-off entre salud y economía, diferentes estrategias han sido seguidas a nivel global. Países como China o Argentina optaron por implementar rápidamente medidas de distanciamiento obligatorio y generalizado, mientras que otros como Estados Unidos o Brasil parecen buscar un mayor equilibrio entre el distanciamiento y la continuidad de la actividad económica. Los consecuencias sociales y económicas se verán en el mediano plazo. En cualquier caso, aquellos países que cuenten con un mayor espacio fiscal tendrán mejores chances de suavizar las consecuencias económicas adversas.

Finalmente, algunas consideraciones deben ser hechas. Las estimaciones aquí reportadas no deben entenderse como una predicción de la dinámica de la pandemia sino, apenas, como un ejercicio de simulación para evaluar la eficacia de las medidas de distanciamiento. Las consecuencias reales de la pandemia podrán ser comprendidas recién en el mediano y largo plazo. En cualquier caso, los gobiernos deben responder activamente con la implementación de testeos considerando una amplia escala poblacional para intentar aproximar en tiempo real la situación epidemiológica.

\section{REFERENCIAS}

1. Johns Hopkins University. Coronavirus COVID-19 global cases. 2020. Disponible en: https://coronavirus.jhu. edu/map.html (Acceso el 3 de agosto de 2020).

2. U.S Bureau of Labor Statistics. Frequently asked questions: The impact of the coronavirus (COVID-19) pandemic on The Employment Situation for March 2020. 2020. Reporte Disponible en: https://www.bls.gov/cps/ employment-situation-covid19-faq-march-2020.pdf

3. Béland L, Wright $\mathrm{T}$, Brodeur A. The Short-Term Economic Consequences of COVID-19: Exposure to Disease, Remote Work and Government Response. 2020. Documento de trabajo 13159, IZA.

4. UNCTAD. Coronavirus: Can policymakers avert a trillion-dollar crisis?. 2020. Reporte publicado en: https://unctad. org/en/pages/newsdetails.aspx?OriginalVersionID =2300
5. Banco Mundial. Proyected poverty impact of COVID-19. 2020. Reporte disponible en: http://pubdocs. worldbank. org/en/461601591649316722/Projected-povertyimpacts-of-COVID-19.pdf

6. van Dorn A, Cooney R, Sabin M. COVID-19 exacerbating inequalities in the U.S. The Lancet. 2020; 395(10232): 1243-1244. DOI: $10.1016 / S 0140-6736(20) 30893-X$

7. European Centre for Disease Prevention and Control. Considerations relating to social distancing measures in response to COVID-19-second update. 2020. Reporte disponible en: https://www.ecdc.europa.eu/sites/ default/files/documents/covid-19-social-distancing-measuresg-guide-second-update.pdf

8. FMI. Policy Responses to COVID-19. 2020a. Disponible en: https://www.imf.org/en/Topics/imf-and-covid19/ Policy-Responses-to-COVID-19\#U

9. Kermack W, McKendrick A. A contribution to the mathematical theory of epidemics. Proceedings of the Royal Society A. 1927; 115: 700-721.

10. Santos M. Pobreza multidimensional en Argentina y Bahía Blanca en tiempos de COVID-19. 2020. Documento de trabajo 14, Instituto de Investigaciones Económicas y Sociales del Sur.

11. Martínez C. Pandemia, situación fiscal argentina y rol de las instituciones en London, S. (comp.) La investigación en ciencias sociales en tiempos de la pandemia por COVID-19. 2020. Documento de trabajo, Instituto de Investigaciones Económicas y Sociales del Sur. Disponible en: https://iiess.conicet.gov.ar/images/DDT/ docTrabajoColectivo2020.pdf

12. González F, Santos M. Las múltiples dimensiones de la pobreza: Posadas en el contexto de la argentina urbana. Visión de Futuro. 2018; 22: 117-136.

13. González F. Regional price dynamics in Argentina (20162019). Regional Statistics. 2020; 10(2): 1-12. DOI: 10.15196/RS100205

14. The Lancet. The gendered dimensions of COVID-19. Nota editorial The Lancet. 2020; 395(10231): 1168. DOI: $10.1016 /$ S0140-6736(20)30823-0

15. Ferguson $N$, Laydon $D$, Nedjati-Gilani G, et al. Impact of non-pharmaceutical interventions (NPIs) to reduce COVID19 mortality and healthcare demand. 2020. Imperial College COVID-19 Response Team

16. Lourenco J, Paton R, Ghafari M et al. Fundamental principles of epidemic spread highlight the immediate need for large-scale serological surveys to assess the stage of the SARS-CoV-2 epidemic. 2020. Disponible en: https://www.medrxiv.org/ content/10.1101/2020.03.24.20042291v1

17. Read J, Bridgen J, Cummings D, Ho A, Jewell C. Novel coronavirus 2019-ncov: early estimation of epidemiological parameters and epidemic predictions. medRxiv. 2020. Disponible en: https://www.medrxiv.org/ content/10.1101/2020.01.23.20018549v2 
18. Remuzzi A, Remuzzi G. Covid-19 and Italy: what next? The Lancet. 2020; 395: 1225-1228.

19. Zhang S, Diao M, Yu W, Pei L, Lin Z, Chen D. Estimation of the reproductive number of novel coronavirus (covid-19) and the probable outbreak size on the diamond princess cruise ship: A data-driven analysis. International Journal of Infectious Diseases. 2020; 93: 201-204. DOI: $10.1016 / j$.ijid.2020.02.033

20. INDEC. Proyecciones poblacionales 2010-2040. 2010. Disponible en: https://www.indec.gob.ar/indec/web/ Nivel4-Tema-2-24-84

21. Google. COVID-19 Community mobility report: Argentina April $11^{\text {th }}, 2020.2020$. Reporte disponible en: https:// www.gstatic.com/covid 19/mobility/2020-04-11_ AR_Mobility_Report_en.pdf

22. Ministerio de Salud de la Nación. Reporte diario coronavirus COVID-19. 2020. Disponible en: https://www. argentina.gob.ar/sites/default/files/02-04-20_reporte-matutino_covid-19.pdf

23. Chudik A, Pesaran M, Rebucci A. Voluntary and Mandatory Social Distancing: Evidence on COVID-19 Exposure Rates from Chinese Provinces and Selected Countries. 2020. Disponible en: https://papers.ssrn. com/sol3/papers.cfm?abstract_id $=3576703$
24. Andersen M. Early Evidence on Social Distancing in Response to COVID-19 in the United States. 2020. Disponible en: https://papers.ssrn.com/sol3/papers. cfm?abstract_id $=3569368$

25. Milne G, Xie S. The Effectiveness of Social Distancing in Mitigating COVID-19 Spread: a modelling analysis. Documento de trabajo. 2020. Disponible en: https://www.medrxiv.org/content/10.1101/2020.03.20.20040055v1.full.pdf

26. Bastos S, Cajueiro D. Modeling and forecasting the early evolution of the Covid-19 pandemic in Brazil. 2020. Disponible en: https://arxiv.org/abs/2003.14288

27. FMI. The Great Lockdown: Worst Economic Downturn Since the Great Depression. 2020b. Disponible en: https://blogs.imf.org/2020/04/14/the-great-lockdown-worst-economic-downturn-since-the-great-depression/

28. Banco Mundial. Serie de población por país. 2018. Reporte disponible en: https://datos.bancomundial.org/ indicador/SP.POP.TOTL

29. Worldometers. Coronavirus Statistics by country. 2020. Disponible en: https://www.worldometers.info/ coronavirus/\# countries 


\section{ANEXO 1}

Los datos referidos a la cantidad de casos confirmados y fallecimientos provienen de lo reportado por la Johns Hopkins University ${ }^{1}$. Esta fuente de información es actualizada diariamente a partir de lo publicado por los respectivos organismos oficiales de cada país. La extracción de datos se realizó empleando una rutina de web-scraping -esto es, en forma automatizada- en STATA $16.1^{* * * * * *}$.

A los efectos de facilitar la comparación entre países se procede a utilizar la cantidad de casos confirmados y fallecimientos relativos a la población:

$$
\begin{gathered}
c_{i}=\frac{C_{i}}{N_{i}} \\
m_{i}=\frac{M_{i}}{N_{i}}
\end{gathered}
$$

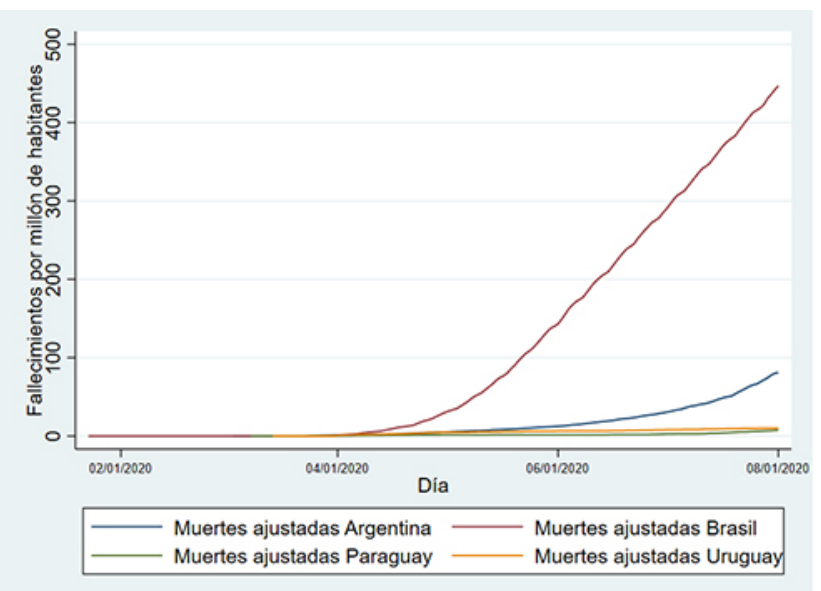

Donde $c_{i}$ es la cantidad de casos confirmados por millón de habitante del país $i$. $C_{i}$ es la cantidad de casos confirmados y $N_{i}$ es la población (en millones de habitantes), del país $i$. Concordantemente, $m_{i}$ y $M_{i}$ refieren a la cantidad de muertes por millón de habitante y cantidad de muertes, respectivamente. Lo referido a cantidad de habitantes surge de las series de población publicadas por el Banco Mundial ${ }^{28}$.

La información referida a la cantidad de test realizados proviene del portal Worldometers ${ }^{29}$. En este caso es relevante la cantidad de test realizados (por millón de habitante) y el promedio diario -desde la detección del primer caso-. Las ecuaciones 3 y 4 presentan estos indicadores:

$$
\begin{gathered}
t_{i}=\frac{T_{i}}{N_{i}} \\
t d_{i}=\frac{T_{i}}{D_{i}}
\end{gathered}
$$

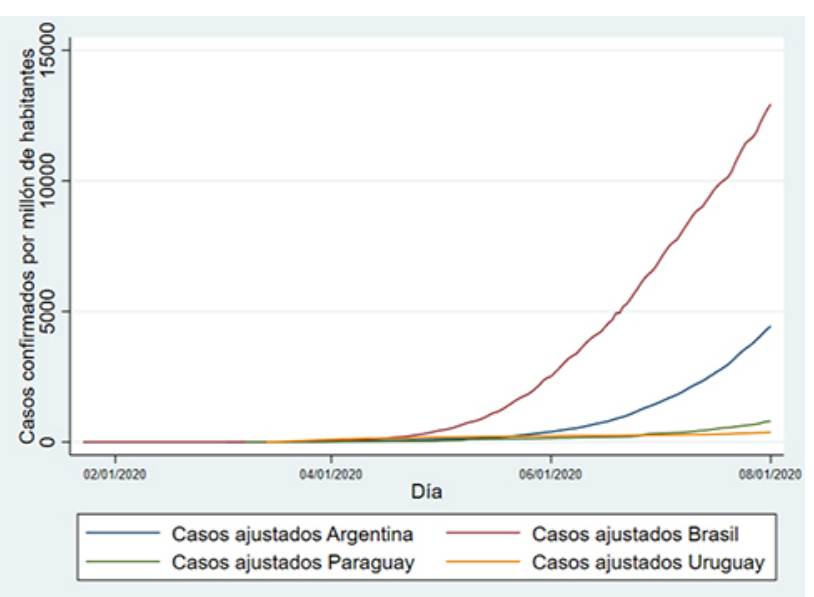

Gráfico 4: Casos confirmados (izquierda) y fallecimientos (derecha) por millón de habitante en el MERCOSUR Fuente: elaboración propia en base a Johns Hopkins University ${ }^{1}$ Nota: se incluyen los registros publicados hasta el 2 de agosto de 2020 inclusive. Escala lineal en ambos casos.

\footnotetext{
$* * * * * *$ La rutina de extracción se encuentra disponible bajo solicitud al autor.
} 

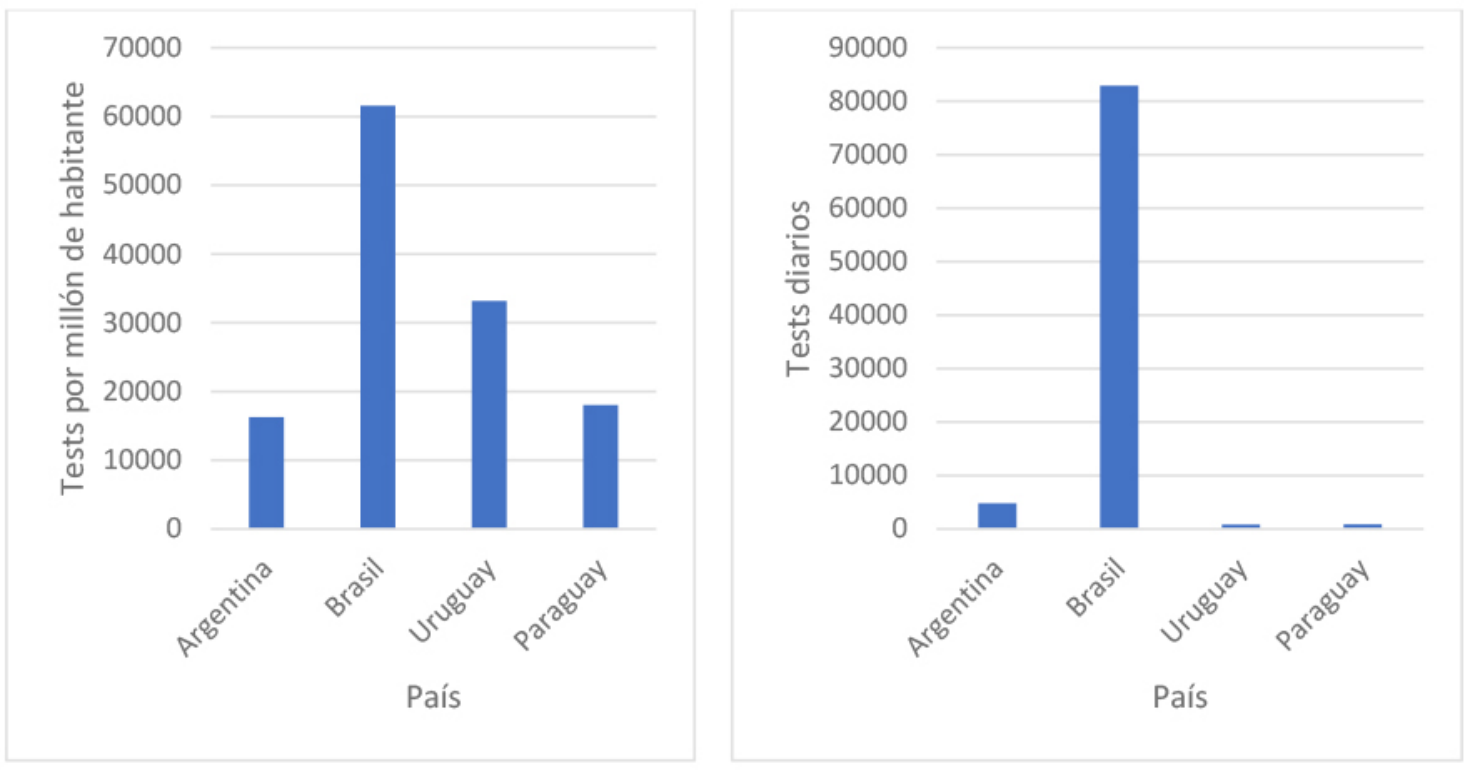

Gráfico 5: Test realizados por millón de habitante (izquierda) y por día (derecha) en el MERCOSUR Fuente: elaboración propia en base a Worldometers ${ }^{29}$

Nota: se incluyen los registros publicados hasta el 2 de agosto de 2020 inclusive. La cantidad de tests diarios se calcula, para cada país, en relación a la cantidad de días transcurridos desde el primer caso confirmado. 